\title{
Quantitative comparison of the magnetic proximity effect in Pt detected by XRMR and XMCD
}

Cite as: Appl. Phys. Lett. 118, 012407 (2021); https://doi.org/10.1063/5.0032584

Submitted: 09 October 2020 . Accepted: 21 December 2020 . Published Online: 06 January 2021

Dominik Graulich, Jan Krieft, Anastasiia Moskaltsova, Johannes Demir, Tobias Peters, Tobias PohImann, (D) Florian Bertram, Joachim Wollschläger, Jose R. L. Mardegan, Sonia Francoual, and (D) Timo Kuschel

(iew Online

\section{ARTICLES YOU MAY BE INTERESTED IN}

Pure spin current phenomena

Applied Physics Letters 117, 190501 (2020); https://doi.org/10.1063/5.0032368

Comparison of measured and simulated spin-wave mode spectra of magnetic nanostructures Applied Physics Letters 118, 012408 (2021); https://doi.org/10.1063/5.0039188

Compositional effect on auto-oscillation behavior of $\mathrm{Ni}_{100-X} \mathrm{Fe}_{\mathbf{X}} / \mathrm{Pt}$ spin Hall nano-oscillators Applied Physics Letters 118, 012406 (2021); https://doi.org/10.1063/5.0035697

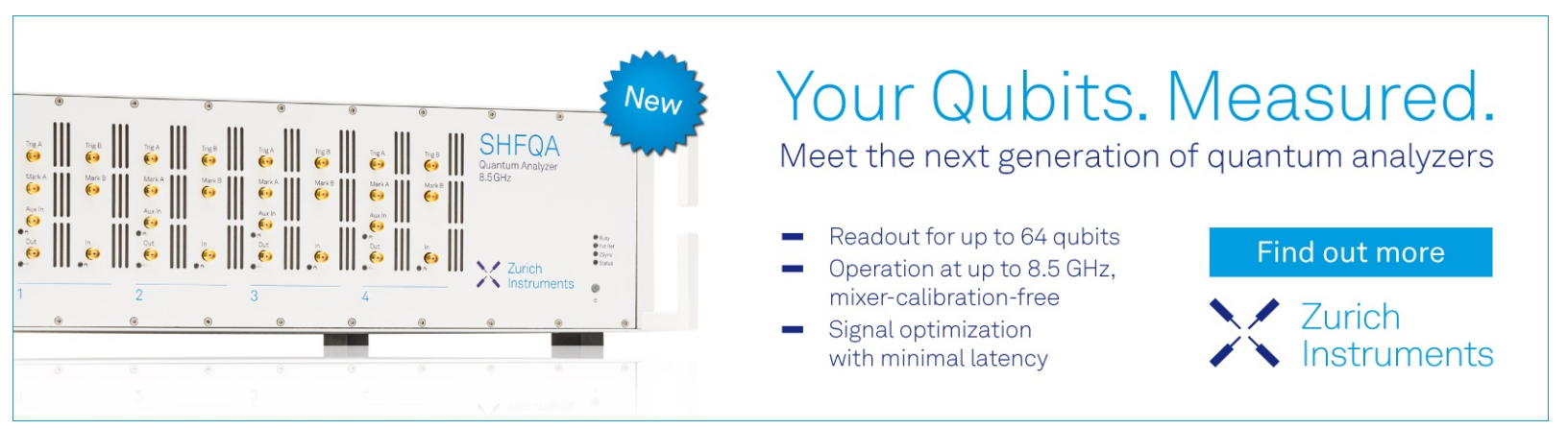




\title{
Quantitative comparison of the magnetic proximity effect in Pt detected by XRMR and XMCD
}

Cite as: Appl. Phys. Lett. 118, 012407 (2021); doi: 10.1063/5.0032584

Submitted: 9 October 2020 - Accepted: 21 December 2020 .

Published Online: 6 January 2021

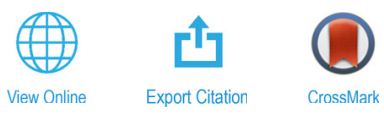

Dominik Graulich, ${ }^{1, a)}$ iD Jan Krieft, ${ }^{\top}$ Anastasiia Moskaltsova, Johannes Demir, Tobias Peters, ${ }^{7}$ Tobias Pohlmann, ${ }^{2,3}$

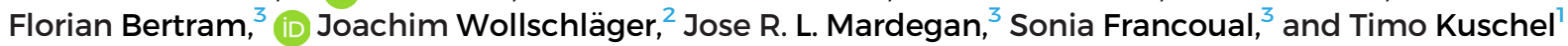

\author{
AFFILIATIONS \\ ${ }^{7}$ Center for Spinelectronic Materials and Devices, Department of Physics, Bielefeld University, Universitätsstraße 25, \\ 33615 Bielefeld, Germany \\ ${ }^{2}$ Center of Physics and Chemistry of New Materials, Department of Physics, Osnabrück University, Barbarastraße 7, \\ 49076 Osnabrück, Germany \\ ${ }^{3}$ Deutsches Elektronen-Synchrotron DESY, Notkestraße 85, 22607 Hamburg, Germany
}

${ }^{a)}$ Author to whom correspondence should be addressed: dgraulich@physik.uni-bielefeld.de

\begin{abstract}
X-ray resonant magnetic reflectivity (XRMR) allows for the simultaneous measurement of structural, optical, and magneto-optic properties and depth profiles of a variety of thin film samples. However, a same-beamtime same-sample systematic quantitative comparison of the magnetic properties observed using XRMR and x-ray magnetic circular dichroism (XMCD) is still pending. Here, the XRMR results (Pt $\mathrm{L}_{3}$ absorption edge) for the magnetic proximity effect in Pt deposited on the two different ferromagnetic materials $\mathrm{Fe}$ and $\mathrm{Co}_{33} \mathrm{Fe}_{67}$ are compared with quantitatively analyzed XMCD results. The obtained results are in very good quantitative agreement between the absorptionbased (XMCD) and reflectivity-based (XRMR) techniques, taking into account an $a b$ initio calculated magneto-optic conversion factor for the XRMR analysis. Thus, it is shown that XRMR provides quantitative reliable spin depth profiles important for spintronic and spin caloritronic transport phenomena at this type of magnetic interfaces.
\end{abstract}

Published under license by AIP Publishing. https://doi.org/10.1063/5.0032584

In the fields of spintronics ${ }^{1}$ and spin caloritronics, ${ }^{2}$ the generation and detection of pure spin currents play an essential role. Here, a common device is a non-magnetic material (NM) thin film used as a spin current detector, which is grown on a ferromagnet (FM). The NM Pt is typically used for the conversion of the spin current into a transverse charge voltage via the inverse spin Hall effect ${ }^{3}$ because of its large spin Hall angle. ${ }^{4}$ For a quantitative analysis, however, one has to take other parasitic effects into account, which can occur due to the closeness of $\mathrm{Pt}$ to the ferromagnetic instability within the Stoner criterion description. ${ }^{5}$ In layered systems of Pt in contact with an FM, the magnetic proximity effect (MPE) can generate a spin-polarized interface within Pt. This can lead to additional effects, e.g., a proximity-induced anomalous Nernst effect in spin Seebeck experiments ${ }^{6,7}$ or a proximityinduced anisotropic magnetoresistance in spin Hall magnetoresistance studies. ${ }^{8}$ It is, therefore, essential to investigate and understand the MPE in systems, which are used for the detection of pure spin currents.
Furthermore, the influence of the MPE on spin-orbit torque (SOT) efficiencies is still under debate. ${ }^{9-11}$ Peterson et al. ${ }^{9}$ report an increase in the field-like SOT by nearly a factor of 4 at $20 \mathrm{~K}$, which is attributed to an increased magnetoresistance caused by the MPE at low temperatures. Contrarily, Zhu et al. ${ }^{10}$ claim the negligible influence of the MPE on SOT efficiencies, whereas an enhancement of the MPE due to annealing was found.

A commonly utilized effect for analyzing the MPE is x-ray magnetic circular dichroism ${ }^{12}$ (XMCD), mostly studied in multilayers of, e.g., Pt adjacent to FMs such as $\mathrm{Fe},{ }^{13} \mathrm{Co},{ }^{14,15}$ and $\mathrm{Ni}^{16}$ Since the $\mathrm{XMCD}$ signal is an average over the whole Pt layer, a thickness variation is usually required to obtain quantitative values for the magnetic moment and the effective thickness of the spin-polarized Pt interface layer ${ }^{17-19}$ using, e.g., fluorescence yield as detection mode, while there are also directly depth-sensitive XMCD implementations on a single sample. ${ }^{20}$ Due to the MPE being an interface effect, a more natural approach for its detection is $\mathrm{x}$-ray resonant magnetic reflectivity ${ }^{21,22}$ 
(XRMR). Here, changes in the magneto-optic absorption $\Delta \beta$ and magneto-optic dispersion $\Delta \delta$ make it directly sensitive to the magnetization density along the normal q-vector and, hence, the magnetic depth profile.

However, additional spectroscopic measurements ${ }^{23}$ or calculations are necessary for a quantitative determination of the spin moments from an XRMR study as compared to XMCD with its sum rules. ${ }^{24-26}$ Usually, the application of the sum rules to experimentally obtained XMCD spectra is necessary to quantify and translate the $\Delta \beta$ spectrum into the induced magnetic moment per atom. Another approach is the application of a magneto-optic conversion factor derived from $a b$ initio calculations, ${ }^{22}$ which has been used for obtaining magnetic moments per Pt atom at the interface from the magnetooptic depth profiles of the XRMR fitting. A systematic comparison of the Pt magnetic moments stemming from a detailed XMCD sum rule analysis and from the magneto-optic depth profiles of XRMR together with the $a b$ initio conversion factor within the same sample systems has not been presented so far.

Therefore, in this manuscript, we tackle a detailed quantitative comparison between the spin magnetic moments measured by the absorption-based (XMCD) and reflectivity-based (XRMR) techniques for two sample stacks consisting of bilayers of Pt (3-4 nm) adjacent to the FMs Fe and $\mathrm{Co}_{33} \mathrm{Fe}_{67}(\sim 10 \mathrm{~nm})$. The samples were prepared by $\mathrm{dc}$ magnetron (co-)sputtering at room temperature from elemental targets onto $\mathrm{MgO}(001)$ substrates. The stoichiometry of the $\mathrm{Co}_{33} \mathrm{Fe}_{67}$ layer was verified by $\mathrm{x}$-ray fluorescence spectroscopy. The first sample, $\mathrm{Pt} / \mathrm{Fe}$, is a standard combination when studying MPE. ${ }^{13,22,27-29}$ The latter sample, $\mathrm{Pt} / \mathrm{Co}_{33} \mathrm{Fe}_{67}$, was chosen due to its high magnetic moment and maximum MPE. ${ }^{30}$ The derived values for the maximum Pt magnetic moments in those studies ${ }^{29,30}$ were $0.5 \pm 0.1 \mu_{\mathrm{B}}$ and $0.72 \pm 0.03 \mu_{\mathrm{B}}$ per Pt atom for $\mathrm{Pt} / \mathrm{Fe}$ and $\mathrm{Pt} / \mathrm{Co}_{33} \mathrm{Fe}_{67}$, respectively, with a typical thickness of $1.1-1.2 \mathrm{~nm}$ for the magnetic Pt layer.

The measurements were carried out at beamline P09 of the third-generation synchrotron at DESY. XRMR was measured in $\theta-$ $2 \theta$ scattering geometry, with a fixed energy and fixed helicity of the circular polarized incident x-rays. The energy was chosen to be $1 \mathrm{eV}$ below $^{14,22,29,31}$ the peak of the $\mathrm{Pt}_{3}$ absorption edge, ${ }^{22}$ called the white line, which was measured by x-ray absorption spectroscopy (XAS) as shown in Fig. 1. The corresponding XMCD measurements were also carried out with a fixed circular polarization of the X-rays. In order to magnetize the samples and generate a magnetic contrast for the measurements, the in-plane magnetic field was switched between parallel and antiparallel orientation with respect to the $\mathrm{x}$-ray beam propagation, with a maximum applied magnetic field of $\pm 150 \mathrm{mT}$. From the two spectra for each edge, $\mathrm{Pt}_{3}$ and $\mathrm{L}_{2}$, the averaged absorption XAS $=\frac{I_{+}+I_{-}}{2}$ and the difference signal defined as $\mathrm{XMCD}=I_{+}-I_{-}$, with the intensity $I_{ \pm}$for positive and negative magnetic fields can be extracted. The measurements were repeated with the opposite $\mathrm{x}$-ray helicity, and the magneto-optic origin was verified by the sign change of the XMCD signal. The magnetic signals obtained for different photon helicities and magnetic field directions were combined to improve the signal-to-noise ratio and remove nonmagnetic artifacts.

In Fig. 1, the measured XAS spectra for both samples together with the $a b$ initio calculated absorption spectrum ${ }^{22}$ are shown. They are relatively shifted in energy to match their peak position and scaled to the absorption coefficient $\beta$ before and after the edge step. Also

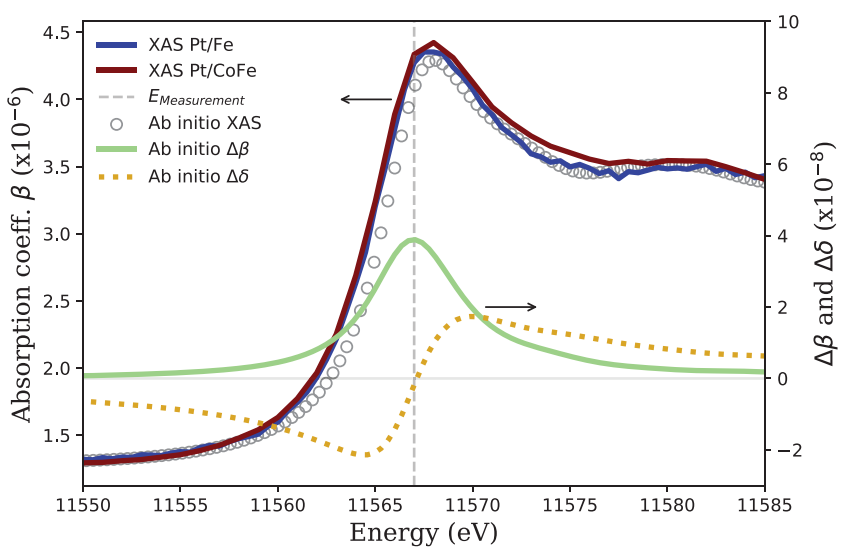

FIG. 1. Calculated ${ }^{22}$ and experimental Pt $L_{3} X A S$ spectra scaled to the absorption coefficient $\beta$ before and after the edge jump. Also shown are the calculated magneto-optic parameters $\Delta \delta$ and $\Delta \beta{ }^{22}$ The calculated spectra were shifted in energy to match the experimental XAS peak positions.

shown is the $a b$ initio calculated magneto-optic parameter $\Delta \beta$, together with $\Delta \delta$, which has been obtained by a Kramers-Kronig transformation of the $\Delta \beta$ spectrum. The incoming x-ray energy used for the XRMR measurements was particularly tuned to $1 \mathrm{eV}$ below the white line peak maximum (gray vertical line in Fig. 1) to be in the maximum of the theoretical $\Delta \beta$ spectrum and without any $\Delta \delta$ contribution, thus reducing the number of fit parameters. The XRMR measurements were also carried out twice, once with each circular polarization, and for each of them, the nonmagnetic x-ray reflectivity (XRR) $I=\frac{I_{+}+I_{-}}{2}$ and the asymmetry ratio $\Delta I=\frac{I_{+}-I_{-}}{I_{+}+I_{-}}$are calculated. Finally, the averages for the two photon helicities and magnetic field directions are calculated to improve data quality. Further information on the two measurement techniques, the experimental details, and the XRMR fitting procedure can be found in the Supplemental Material (including Refs. 32-38).

Figures 2(a) and 2(b) present the XRR curve and the XRMR asymmetry ratio, respectively, for both samples. For fitting the data, the absorption $\beta$ of the Pt layer was taken from the XAS scans in Fig. 1 since this parameter showed to have the largest influence on the quantitative value of the resulting induced magnetic moments. ${ }^{28}$ Having the structural and optical parameters fixed from fitting the XRR curves, ${ }^{22,28}$ the asymmetry ratio signal was modeled by the variation of a magneto-optic depth profile. Since the energy was chosen to minimize the influence of $\Delta \delta$ (see the supplementary material for further information), only the spatial distribution and amplitude of $\Delta \beta$ were varied during fitting $\Delta I$. The modeled Gaussian depth profiles of $\Delta \beta$ were convoluted with the roughnesses of the Pt/FM interfaces, resulting in the Gaussian-like depth profiles shown in Fig. 2(c), which correspond to the asymmetry ratio fits in Fig. 2(b). The $\Delta \beta$ depth profile was converted into the induced Pt moment by the $a b$ initio calculated conversion factor [see Fig. 2(c)], and for the maximum Pt moment, the peak value of the Gaussian-like depth profile was taken.

For the $\mathrm{Pt} / \mathrm{Fe}$ sample, we obtain a maximum spin magnetic moment of $0.47 \mu_{\mathrm{B}}$ per Pt atom, which is in good agreement with the values previously reported for similar Pt/Fe samples. ${ }^{28,29}$ Although a similar moment was found, the full width at half maximum (FWHM), an indication for the extension of the spin polarization, is slightly 

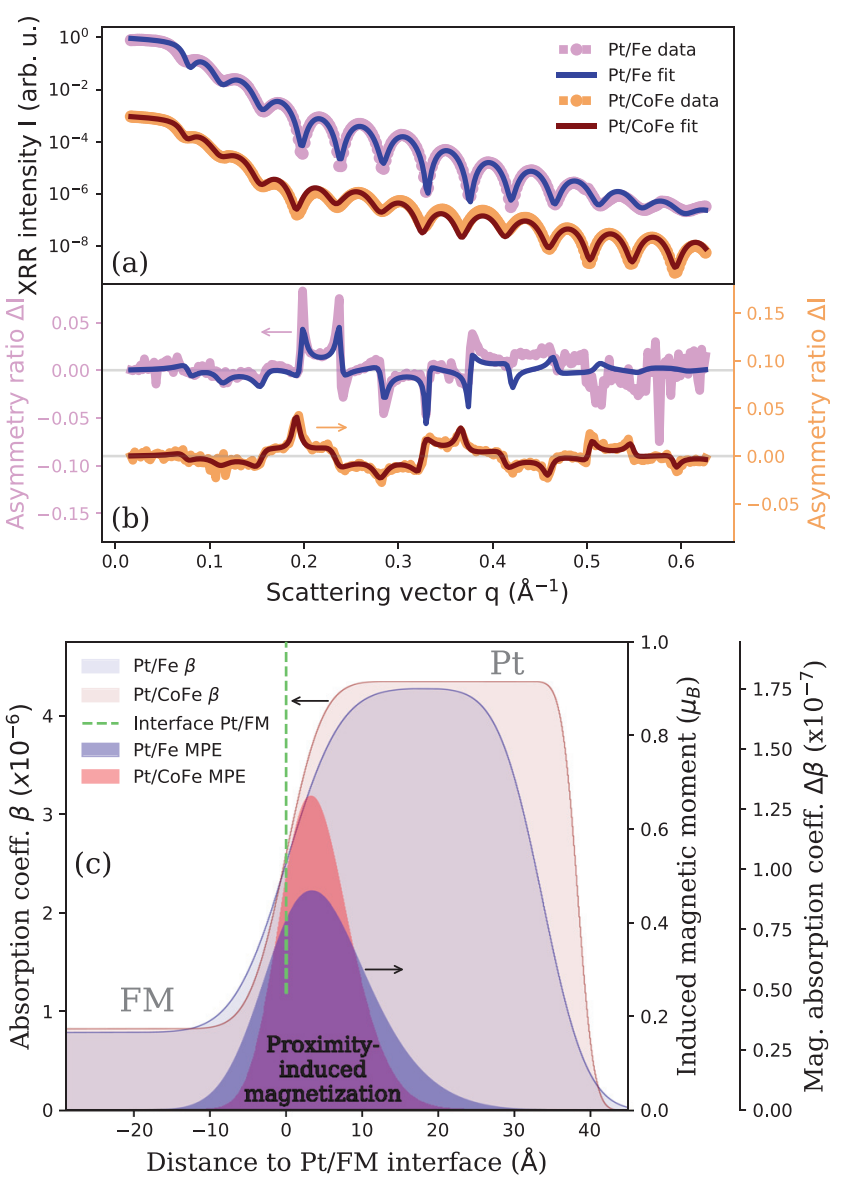

FIG. 2. (a) Data and fits of the averaged resonant XRR curves. (b) Resulting asymmetry ratios calculated from the normalized difference of the two measurements with opposite $\mathrm{x}$-ray helicity. (c) Modeled magneto-optic depth profiles of $\Delta \beta$ together with the derived induced magnetic moment for the two samples (conversion factor taken as $\mu_{\text {spin }}^{\mathrm{Pt}}=\Delta \beta \cdot 5.14 \times 10^{6} \mu_{\mathrm{B}} / \mathrm{atom}^{22}$ ). The optical depth profiles obtained from the resonant XRR fit are also shown.

larger in the present work. We find an FWHM of $1.6 \mathrm{~nm}$ within the $3.4 \mathrm{~nm}$ Pt layer, whereas previously reported values are $1.1-1.2 \mathrm{~nm}$. This can be explained by a rather large roughness. When compared to the previously reported range of $0.45 \pm 0.10 \mathrm{~nm}$ for the interfacial roughness for this kind of sample, $0.55 \mathrm{~nm}$ at the $\mathrm{Pt} / \mathrm{Fe}$ interface of the present work is rather large. This probably results in a stronger intermixing of $\mathrm{Pt}$ and $\mathrm{Fe}$ and, therefore, a wider spin polarized Pt layer. For the $\mathrm{Pt} / \mathrm{Co}_{33} \mathrm{Fe}_{67}$ sample, we obtain a maximum value for the spin magnetic moment of $0.67 \mu_{\mathrm{B}}$ per Pt atom with an FWHM of $1 \mathrm{~nm}$ within a $3.8 \mathrm{~nm}$ Pt layer. Here, the roughness is smaller being $0.38 \mathrm{~nm}$, also indicating the interplay of interfacial roughness and spread of the proximity-induced magnetization. These quantitative XRMR results are summarized in Table I. For both samples, only the spin contribution to the Pt magnetization is considered.

Since the asymmetry ratio data of the $\mathrm{Pt} / \mathrm{Co}_{33} \mathrm{Fe}_{67}$ sample are better reproduced by the fit than for the $\mathrm{Pt} / \mathrm{Fe}$ sample, especially after $\mathrm{q}=0.4 \AA^{-1}$, alterations from the Gaussian model for the depth profile have been considered. However, the fit does not improve to a
TABLE I. Results from XRMR (including $\Delta \beta$-to-magnetic moment conversion) and $X M C D$ sum rule analysis. Displayed are the thickness of the Pt layer $t_{P t}$, the FWHM of the magnetic part of Pt $t_{\mathrm{MPE}}$, and the interfacial roughness $\sigma_{\mathrm{Pt} / \mathrm{FM}}$ as obtained from XRMR. The XMCD sum rule analysis yields the spin moment $m_{\mathrm{spin}}^{\mathrm{XMCD}}$ and an upper limit for the orbital moment $m_{\text {orb }}^{\mathrm{XMCD} \text {,lim }}$. The value $m_{\text {spin }}^{\mathrm{XRMR}}$ is derived from the XRMR analysis using the $a b$ initio conversion factor, while $m_{\text {spin }}^{\mathrm{XMC} \text {,scaled }}$ is obtained by using the XMCD result for the scaling of the $\Delta \beta$ depth profile.

\begin{tabular}{lcc}
\hline \hline & $\mathrm{Pt} / \mathrm{Fe}$ & $\mathrm{Pt} / \mathrm{Co}_{33} \mathrm{Fe}_{67}$ \\
\hline$t_{\mathrm{Pt}}$ & $3.37 \pm 0.05 \mathrm{~nm}$ & $3.84 \pm 0.05 \mathrm{~nm}$ \\
$t_{\mathrm{MPE}}$ & $1.56 \pm 0.10 \mathrm{~nm}$ & $1.04 \pm 0.10 \mathrm{~nm}$ \\
$\sigma_{\mathrm{Pt} / \mathrm{FM}}$ & $0.55 \pm 0.05 \mathrm{~nm}$ & $0.38 \pm 0.05 \mathrm{~nm}$ \\
$m_{\text {orb }}^{\mathrm{XMCD}}$ iim & $<0.05 \mu_{\mathrm{B}}$ & $<0.015 \mu_{\mathrm{B}}$ \\
$m_{\text {spin }}^{\mathrm{XMCD}}$ & $0.22 \pm 0.05 \mu_{\mathrm{B}}$ & $0.18 \pm 0.04 \mu_{\mathrm{B}}$ \\
$m_{\text {spin }}^{\mathrm{XRMR}}$ & $0.47 \pm 0.10 \mu_{\mathrm{B}}$ & $0.67 \pm 0.10 \mu_{\mathrm{B}}$ \\
$m_{\text {spin }}^{\mathrm{XMC}, \text { scaled }}$ & $0.45 \pm 0.14 \mu_{\mathrm{B}}$ & $0.63 \pm 0.21 \mu_{\mathrm{B}}$ \\
\hline \hline
\end{tabular}

significant extent and the values at maximum as well as the FWHMs of the resulting $\Delta \beta$ profiles are within the given error range for that sample.

The XAS and XMCD spectra are presented in Fig. 3. A clear dichroism can be seen for both samples at the $\mathrm{Pt} \mathrm{L}_{3}$ and $\mathrm{L}_{2}$ edges. For the determination of the orbital and spin moments, the sum rules ${ }^{24-26}$ were applied. As part of the data postprocessing, the pre-edge slope of the XAS spectra was corrected by a linear fit and the spectra were normalized to an $\mathrm{L}_{3}-\mathrm{L}_{2}$ edge jump ratio of $R=2.22$, as experimentally found for pure $\mathrm{Pt}^{39}$ For the removal of the continuum contribution, an experimentally obtained $\mathrm{Au}$ reference spectrum has been subtracted, following the procedure described in Ref. 40. Due to the generally small white line of $\mathrm{Pt}$, the alternative approach of subtracting a step function, as typically done in the soft $\mathrm{x}$-ray range, would lead to a large uncertainty contribution in the determination of the spin and orbital moments. $^{41}$

XAS spectra of $0.2-\mu \mathrm{m}$-thick $\mathrm{Au}$ and $\mathrm{Pt}\left(\mathrm{Pt}_{\mathrm{ref}}\right)$ films, resembling the metallic state in terms of $5 \mathrm{~d}$ holes, have been collected. The Au spectrum has been stretched and shifted in energy to match the extended $\mathrm{x}$-ray absorption fine structure features of the $\mathrm{Pt}_{\text {ref }}$ spectrum. By matching the near edge features of the $\mathrm{Pt}_{\text {ref }}$ measurement to the $\mathrm{Pt} / \mathrm{FM}$ samples, accounting for small variations in energy of the different measurements, the $\mathrm{Au}$ spectrum has been scaled as shown in Fig. 3. The difference between the XAS of the Pt/FM and the Au reference, called $r_{\mathrm{Pt} / \mathrm{FM}}$, has been calculated, as well as the difference between the XAS of $\mathrm{Pt}_{\text {ref }}$ and of the Au reference $\left(r_{\mathrm{Pt} \text {,ref }}\right)$. The subtraction of the Au reference from $\mathrm{Pt}_{\text {ref }}$ with the relative difference in $5 \mathrm{~d}$ holes ${ }^{18,40}$ of $n_{\mathrm{h}}^{\text {dif }}=1.06\left(n_{\mathrm{h}}^{\text {Pt,ref }}=1.80\right.$ and $\left.n_{\mathrm{h}}^{\text {Au }}=0.74\right)$ yields a scaling factor, which can be used to determine the number of holes for the Pt/FM samples: ${ }^{42} n_{\mathrm{h}}^{\mathrm{Pt} / \mathrm{FM}}=n_{\mathrm{h}}^{\mathrm{Au}}+r_{\mathrm{Pt} / \mathrm{FM}} n_{\mathrm{h}}^{\text {dif }} / r_{\mathrm{Pt} \text {,ref }}$.

With the integration of the $\mathrm{L}_{3} \mathrm{XMCD}$ signal $p$ and the total integrated XMCD signal, $\mathrm{L}_{3}+\mathrm{L}_{2}, q$, the sum rules can be written as

$$
m_{\mathrm{orb}}=\frac{2}{3} n_{\mathrm{h}}^{\mathrm{Pt} / \mathrm{FM}} \frac{q}{r_{\mathrm{Pt} / \mathrm{FM}}} \quad ; \quad m_{\mathrm{spin}}=n_{\mathrm{h}}^{\mathrm{Pt} / \mathrm{FM}} \frac{(3 p-2 q)}{r_{\mathrm{Pt} / \mathrm{FM}}} .
$$

The results can be found in Table I. The procedure for the determination of the error bars for both methods can be found in the 

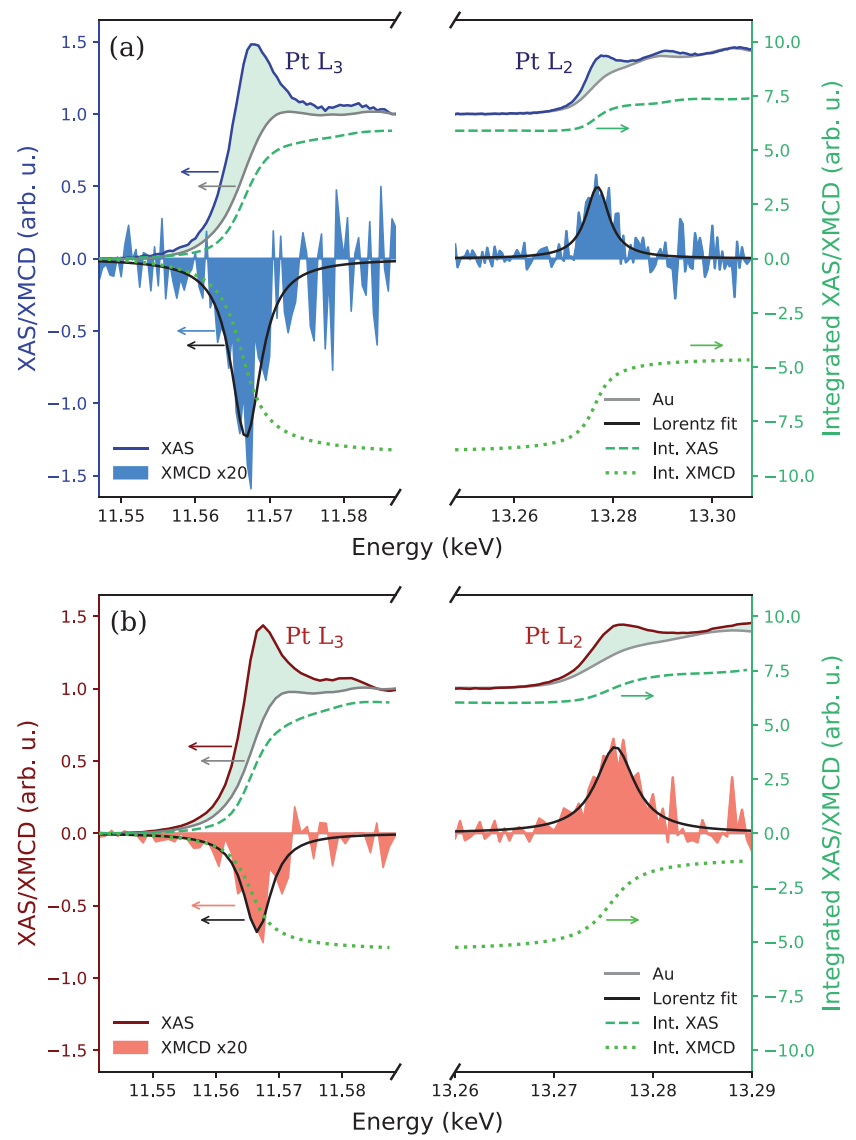

FIG. 3. Experimental XAS for (a) the Pt/Fe sample and (b) the $\mathrm{Pt} / \mathrm{CO}_{33} \mathrm{Fe}_{67}$ sample at the $L$ absorption edges of Pt. The XAS data are averaged over the two $x$-ray helicities and magnetic field directions, and the XMCD signal is scaled by a factor of 20 (filled area). Additionally shown is the Au reference absorption spectrum, shifted as described in the text. The integration of the difference between the Pt/FM XAS signal and the Au XAS as well as the integration of the XMCD signal are shown as dotted lines, for the XAS additionally indicated as the shaded area. The XMCD signal has been fitted with a Lorentzian to reduce the noise while integrating.

supplementary material (including Ref. 43) together with a discussion on the orbital moments, which are neglected in the following analysis.

While the stoichiometry of the FM in the $\mathrm{Pt} / \mathrm{Co}_{33} \mathrm{Fe}_{67}$ sample was chosen to have the largest spin moment as shown in a previous $\mathrm{XRMR}$ study, ${ }^{30}$ the obtained value from the XMCD analysis is smaller when compared to the $\mathrm{Pt} / \mathrm{Fe}$ sample. This can be explained by looking at the results from the XRMR analysis displayed in Table I. The Pt layer is slightly thicker for the $\mathrm{Pt} / \mathrm{Co}_{33} \mathrm{Fe}_{67}$ sample, and the FWHM of the spin polarized $\mathrm{Pt}$ is only two thirds of the value for the $\mathrm{Pt} / \mathrm{Fe}$ sample. This coincides with the larger interfacial roughness of the latter sample. The obtained magnetic moments are relatively suppressed by the non-magnetic $\mathrm{Pt}$, thus contributing less to the XMCD signal. Without a thickness variation of $\mathrm{Pt}$, the quantitative values derived from the XMCD sum rule analysis give no information about the distribution of the magnetic atoms in the case of induced magnetism at the interface over a very small depth.

The magnetic moment from the XMCD analysis gives an averaged moment over the complete Pt layer, while the XRMR depth profile considers a larger but at the Pt/FM interface located magnetization. For the quantitative comparison between the two x-ray techniques, we can compare the integrated area of the $\Delta \beta$ depth profile, scaled by the $a b$ initio conversion factor, with the area of the total $\mathrm{Pt}$ depth profile, scaled to the XMCD spin moment. The differences are $4 \%$ and $6 \%$ for $\mathrm{Pt} / \mathrm{Fe}$ and $\mathrm{Pt} / \mathrm{Co}_{33} \mathrm{Fe}_{67}$, respectively, with the XRMR result being slightly larger for both samples. Furthermore, we can consider the spin magnetic moments obtained from the XRMR $\Delta \beta$ depth profile, when using the XMCD results instead of the $a b$ initio calculation for the conversion to units of $\mu_{\mathrm{B}}$. This would yield maximum spin magnetic moments of $0.45 \pm 0.14 \mu_{\mathrm{B}}$ and $0.63 \pm 0.22 \mu_{\mathrm{B}}$ for $\mathrm{Pt} / \mathrm{Fe}$ and $\mathrm{Pt} / \mathrm{Co}_{33} \mathrm{Fe}_{67}$, respectively. Despite the error bars being rather large, the obtained spin moment values are still in very good agreement, showing the quantitative reliability of the $a b$ initio conversion factor. Further information on these calculations can be found in the Supplemental Material.

Being the first comparison of this kind, we can only call for further such multi-modal studies of interfacial magnetism, both for systematic quantitative checks of consistency between XRMR and $\mathrm{XMCD}$ and for the inherent complementarity of these two probes.

For samples with very high interfacial roughness or nonconducting FMs, where no MPE is expected and its presence is caused by interdiffusion due to the sample preparation, the differences in the quantitative results seem to get larger as found for the inverted $\mathrm{Y}_{3} \mathrm{Fe}_{5} \mathrm{O}_{12} / \mathrm{Pt}$ structure. ${ }^{44}$ This could be caused by the change of the atomic surrounding of $\mathrm{Pt}$, limiting the applicability of the calculated ab initio conversion factor or sum rule analysis, or other factors, which cannot be described by at least one of the two techniques without further investigations.

In conclusion, it was shown in this work that the quantitative results of the XRMR analysis, including the $\Delta \beta$-magnetic moment conversion factor, are very much comparable to the ones obtained by $\mathrm{XMCD}$ and a sum rule analysis for the given kind of sample. While XRMR and XMCD are complementary methods and a multi-modal approach is surely favorable in the case of unknown sample types, it has been shown that for the given sample type, XRMR can be used as a stand-alone quantitative method. Provided that an ab initio factor can be calculated and has been confirmed, XRMR can be used in the analysis of magnetic depth profiles, as shown here for Pt and samples involving low interfacial roughness, without the need of conducting additional XMCD measurements to obtain reliable results. The increase in magnetization in the NM due to the MPE must be known for a quantitative analysis of phenomena such as SOT efficiencies and spin Hall angle measurements. Specifically, when comparing samples with slight variations in the thickness of the NM and/or the spin polarized layer (e.g., due to different growth conditions) or samples with more than one interface of interest (e.g., trilayers with asymmetric $\mathrm{MPE}^{45,46}$ ), any $\mathrm{XMCD}$ result, for instance, can only reveal changes in the overall averaged magnetic moment value and not the differences in the magnetic moment distribution. Exactly the former case has been highlighted in our presented study. However, this information, the spatial distribution of magnetic moments and its quantification, is now available and confirmed using XRMR in combination with the reliable $\Delta \beta$-magnetic moment conversion factor for $\mathrm{Pt}$, which is $\mu_{\text {spin }}^{\mathrm{Pt}}=\Delta \beta \cdot 5.14 \times 10^{6} \mu_{\mathrm{B}} /$ atom.

See the supplementary material for additional information about the measurement techniques, the XRMR fitting procedure, 
calculations for the quantitative comparison of the XRMR and XMCD results, the determination of the error bars, Kramers-Kronig transformation of the XMCD signal, and a discussion on the orbital moments as obtained from the XMCD sum rule analysis.

We acknowledge DESY (Hamburg, Germany), a member of the Helmholtz Association HGF, for the provision of experimental facilities. Parts of this research were carried out at beamline P09 at PETRA III. We would like to thank Günter Reiss for making available the laboratory equipment in Bielefeld and acknowledge financial support from the Deutsche Forschungsgemeinschaft (DFG) within Grant No. RE 1052/42-1.

\section{DATA AVAILABILITY}

The data that support the findings of this study are available from the corresponding author upon reasonable request.

\section{REFERENCES}

${ }^{1}$ S. A. Wolf, D. D. Awschalom, R. A. Buhrman, J. M. Daughton, S. Von Molnár, M. L. Roukes, A. Y. Chtchelkanova, and D. M. Treger, Science 294, 1488 (2001).

${ }^{2}$ G. E. Bauer, E. Saitoh, and B. J. Van Wees, Nat. Mater. 11, 391 (2012).

${ }^{3}$ E. Saitoh, M. Ueda, H. Miyajima, and G. Tatara, Appl. Phys. Lett. 88, 182509 (2006).

${ }^{4}$ A. Hoffmann, IEEE Trans. Magn. 49, 5172 (2013).

${ }^{5}$ E. C. Stoner, Proc. R. Soc. London. Ser. A. Math. Phys. Sci. 165, 372 (1938).

${ }^{6}$ S. Y. Huang, X. Fan, D. Qu, Y. P. Chen, W. G. Wang, J. Wu, T. Y. Chen, J. Q. Xiao, and C. L. Chien, Phys. Rev. Lett. 109, 107204 (2012).

${ }^{7}$ P. Bougiatioti, C. Klewe, D. Meier, O. Manos, O. Kuschel, J. Wollschläger, L. Bouchenoire, S. D. Brown, J. M. Schmalhorst, G. Reiss, and T. Kuschel, Phys. Rev. Lett. 119, 227205 (2017).

${ }^{8}$ M. Althammer, S. Meyer, H. Nakayama, M. Schreier, S. Altmannshofer, M. Weiler, H. Huebl, S. Geprägs, M. Opel, R. Gross, D. Meier, C. Klewe, T. Kuschel, J. M. Schmalhorst, G. Reiss, L. Shen, A. Gupta, Y. T. Chen, G. E. W. Bauer, S. T. B. Saitoh, and E. Goennenwein, Phys. Rev. B 87, 224401 (2013).

${ }^{9}$ T. A. Peterson, A. P. McFadden, C. J. Palmstrøm, and P. A. Crowell, Phys. Rev. B 97, 020403 (2018).

${ }^{10}$ L. J. Zhu, D. C. Ralph, and R. A. Buhrman, Phys. Rev. B 98, 134406 (2018).

${ }^{11}$ A. Mostkaltsova, J. Krieft, D. Graulich, T. Matalla-Wanger, and T. Kuschel, AIP Adv. 10, 015154 (2020)

${ }^{12}$ G. Schütz, W. Wagner, W. Wilhelm, P. Kienle, R. Zeller, R. Frahm, and G. Materlik, Phys. Rev. Lett. 58, 737 (1987).

${ }^{13}$ W. J. Antel, Jr., M. M. Schwickert, T. Lin, W. L. O’Brien, and G. R. Harp, Phys. Rev. B 60, 12933 (1999).

${ }^{14}$ G. Schütz, R. Wienke, W. Wilhelm, W. B. Zeper, H. Ebert, and K. Spörl, J. Appl. Phys. 67, 4456 (1990).

${ }^{15}$ F. Wilhelm, P. Poulopoulos, A. Scherz, H. Wende, K. Baberschke, M. Angelakeris, N. K. Flevaris, J. Goulon, and A. Rogalev, Phys. Status Solidi 196, 33 (2003).

${ }^{16}$ F. Wilhelm, P. Poulopoulos, G. Ceballos, H. Wende, K. Baberschke, P. Srivastava, D. Benea, H. Ebert, M. Angelakeris, N. K. Flevaris, D. Niarchos, A. Rogalev, and N. B. Brookes, Phys. Rev. Lett. 85, 413 (2000).

${ }^{17}$ S. Rüegg, G. Schütz, P. Fischer, R. Wienke, W. B. Zeper, and H. Ebert, J. Appl. Phys. 69, 5655 (1991).

${ }^{18}$ P. Poulopoulos, F. Wilhelm, H. Wende, G. Ceballos, K. Baberschke, D. Benea, H. Ebert, M. Angelakeris, N. K. Flevaris, A. Rogalev, and N. B. Brookes, J. Appl. Phys. 89, 3874 (2001).
${ }^{19}$ M. Suzuki, H. Muraoka, Y. Inaba, N. Miyagawa, H. Kawamura, T. Shimatsu, H. Maruyama, N. Ishimatsu, Y. Isohama, and Y. Sonobe, Phys. Rev. B 72, 054430 (2005).

${ }^{20}$ K. Amemiya, S. Kitagawa, D. Matsumura, T. Yokoyama, and T. Ohta, J. Phys.: Condens. Matter 15, S561 (2003).

${ }^{21}$ S. Macke and E. Goering, J. Phys.: Condens. Matter 26, 363201 (2014).

${ }^{22}$ T. Kuschel, C. Klewe, J. M. Schmalhorst, F. Bertram, O. Kuschel, T. Schemme, J. Wollschläger, S. Francoual, J. Strempfer, A. Gupta, M. Meinert, G. Götz, D. Meier, and G. Reiss, Phys. Rev. Lett. 115, 097401 (2015).

${ }^{23}$ L. Sève, N. Jaouen, J. M. Tonnerre, D. Raoux, F. Bartolomé, M. Arend, W. Felsch, A. Rogalev, J. Goulon, C. Gautier, and J. F. Bérar, Phys. Rev. B 60, 9662 (1999).

${ }^{24}$ B. T. Thole, P. Carra, F. Sette, and G. Van Der Laan, Phys. Rev. Lett. 68, 1943 (1992).

${ }^{25}$ P. Carra, B. T. Thole, M. Altarelli, and X. Wang, Phys. Rev. Lett. 70, 694 (1993).

${ }^{26}$ C. T. Chen, Y. U. Idzerda, H.-J. Lin, N. V. Smith, G. Meigs, E. Chaban, G. H. Ho, E. Pellegrin, and F. Sette, Phys. Rev. Lett. 75, 152 (1995).

${ }^{27}$ S. Geprägs, S. Meyer, S. Altmannshofer, M. Opel, F. Wilhelm, A. Rogalev, R. Gross, and S. T. B. Goennenwein, Apl. Phys. Lett. 101, 262407 (2012).

${ }^{28}$ C. Klewe, T. Kuschel, J.-M. Schmalhorst, F. Bertram, O. Kuschel, J. Wollschläger, J. Strempfer, M. Meinert, and G. Reiss, Phys. Rev. B 93, 214440 (2016).

${ }^{29}$ T. Kuschel, C. Klewe, P. Bougiatioti, O. Kuschel, J. Wollschläger, L. Bouchenoire, S. D. Brown, J.-M. Schmalhorst, D. Meier, and G. Reiss, IEEE Trans. Magn. 52, 1 (2016).

${ }^{30} \mathrm{P}$. Bougiatioti, O. Manos, O. Kuschel, M. Tolkiehn, S. Francoual, and T. Kuschel, arXiv:1807.09032 (2018).

${ }^{31}$ J. Geissler, E. Goering, M. Justen, F. Weigand, G. Schütz, J. Langer, D. Schmitz, H. Maletta, and R. Mattheis, Phys. Rev. B 65, 020405(R) (2001).

${ }^{32}$ J. Strempfer, S. Francoual, D. Reuther, D. K. Shukla, A. Skaugen, H. SchulteSchrepping, T. Kracht, and H. Franz, J. Synchrotron Radiat. 20, 541 (2013).

${ }^{33}$ S. Macke, A. Radi, J. E. Hamann-Borrero, A. Verna, M. Bluschke, S. Brück, E. Goering, R. Sutarto, F. He, G. Cristiani, M. Wu, E. Benckiser, H.-U. Habermeier, G. Logvenov, N. Gauquelin, G. A. Botton, A. P. Kajdos, S. Stemmer, G. A. Sawatzky, M. W. Haverkort, B. Keimer, and V. Hinkov, Adv. Mater. 26, 6554 (2014).

${ }^{34}$ L. G. Parratt, Phys. Rev. 95, 359 (1954).

${ }^{35}$ L. Névot and P. Croce, Rev. Phys. Appl. 15, 761 (1980).

${ }^{36}$ J. Zak, E. R. Moog, C. Liu, and S. D. Bader, J. Magn. Magn. Mater. 89, 107 (1990).

${ }^{37}$ B. L. Henke, E. M. Gullikson, and J. C. Davis, At. Data Nucl. Data Tables 54, 181 (1993).

${ }^{38}$ J. Krieft, D. Graulich, A. Moskaltsova, L. Bouchenoire, S. Francoual, and T. Kuschel, J. Phys. D: Appl. Phys. 53, 375004 (2020).

${ }^{39}$ L. F. Mattheiss and R. E. Dietz, Phys. Rev. B 22, 1663 (1980).

${ }^{40}$ W. Grange, M. Maret, J. P. Kappler, J. Vogel, and A. Fontaine, Phys. Rev. B 58, 6298 (1998).

${ }^{41}$ F. Wilhelm, P. Poulopoulos, H. Wende, A. Scherz, K. Baberschke, M. Angelakeris, N. K. Flevaris, and A. Rogalev, Phys. Rev. Lett. 87, 207202 (2001).

${ }^{42}$ J. Vogel, A. Fontaine, V. Cros, and F. Petroff, Phys. Rev. B 55, 3663 (1997).

${ }^{43}$ M. A. W. Schoen, J. Lucassen, H. T. Nembach, T. J. Silva, B. Koopmans, C. H. Back, and J. M. Shaw, Phys. Rev. B 95, 134410 (2017).

${ }^{44}$ S. Geprägs, C. Klewe, S. Meyer, D. Graulich, F. Schade, M. Schneider, S. Francoual, S. P. Collins, K. Ollefs, F. Wilhelm, A. Rogalev, Y. Joly, S. T. B. Goennenwein, M. Opel, T. Kuschel, and R. Gross, Phys. Rev. B 102, 214438 (2020).

${ }^{45}$ O. Inyang, L. Bouchenoire, B. Nicholson, M. Tokaç, R. M. Rowan-Robinson, C. J. Kinane, and A. T. Hindmarch, Phys. Rev. B 100, 174418 (2019).

${ }^{46}$ A. Mukhopadhyay, S. K. Vayali, D. Graulich, I. Ahamed, S. Francoual, A. Kashyap, T. Kuschel, and P. S. A. Kumar, Phys. Rev. B 102, 094309 (2020). 